\title{
Dynamics and control of foot-and-mouth disease in endemic countries: a pair approximation model
}

\author{
N. Ringa ${ }^{1}$, C.T. Bauch ${ }^{1,2}$ \\ ${ }^{1}$ Department of Mathematics and Statistics, University of Guelph, 50 Stone Rd E, Guelph, ON N1G \\ 2W1, Canada, ${ }^{2}$ Department of Applied Mathematics, University of Waterloo, 200 University Avenue \\ West Waterloo, ON N2L 3G1, Canada.
}

\begin{abstract}
Various mathematical models have been developed to describe spatial farm-to-farm transmission of foot and mouth disease (FMD). These models have explored the impacts of control measures such as culling and vaccination during a single outbreak in a country normally free of FMD. As a result, these models do not include factors that are relevant to countries where FMD is endemic in some regions, like long-term waning natural and vaccine immunity, use of prophylactic vaccination, and disease re-importations. These factors may have implications for disease dynamics and control, and yet few mathematical models have been developed for FMD-endemic settings. Here we develop and study an SEIRV (susceptible-exposed-infectious-recovered-vaccinated) pair approximation model of FMD. We focus on long term dynamics by exploring characteristics of repeated outbreaks of FMD and their dependence on disease re-importation, loss of natural immunity, and vaccine waning. We find that prophylactic vaccination is more effective than ring vaccination, for the same per capita vaccination rate, but the effectiveness of any vaccination strategy strongly depends on duration of natural immunity, rate of vaccine waning, and disease re-introduction. Changing the duration of natural immunity generally has a more profound impact on the number and magnitudes of FMD outbreaks than changing the duration of vaccine immunity. If loss of natural immunity and/or vaccine waning happen rapidly, then large multiple subsequent outbreaks result, making it difficult to eliminate the disease. Finally, more frequent disease re-importations causes a higher cumulative number of infections, although a lower average epidemic peak. Because of the differences between dynamics in FMD-free settings versus FMD-endemic settings, and because FMD is a global problem, more mathematical models tailored to FMD-endemic countries should be developed that include factors like waning natural and vaccine immunity and the impact of repeated disease importations.
\end{abstract}

Keywords: foot-and-mouth disease, pair approximation models, prophylactic vaccination, ring vaccination, natural immunity. 


\section{Introduction}

Foot and mouth disease (FMD) is a highly transmissible viral infection affecting cloven-hoofed animals, including domestic livestock such as cattle, pigs, goats, sheep [3, 24, 40, 18] and some wild animals, e.g. buffaloes. The disease agent of FMD belongs to the picorna virus family [7]. There are seven known serotypes of FMD virus which vary according to geographical region $[35,1]$. The serotypes of FMD are classified as: (a) European types O, A and C; (b) African types STA 1, STA 2 and STA 3 and (c) Asian type Asia 1 $[12,13]$, and there are several (more than $60)$ subtypes of the virus $[1,2,7]$. Vaccination against one serotype does not provide protection against other serotypes. This makes it makes it difficult to control the spread of FMD by vaccination alone, and adopting multiple control measures may offer better means of control.

The FMD virus can be found in secretions and excretions from infected animals, including expired air, saliva, milk, urine and semen. The virus is airborne and can also transmitted through physical contact. Clinical symptoms of FMD include high fever, blisters inside the mouth and stunted growth [35, 3, 18, 34]. However, animals rarely die from foot and mouth disease. Upon introduction, FMD virus spreads rapidly within a farm, and interactions between neighboring farms lead to a rapid spread of the disease to several kilometers (up to $6 \mathrm{~km}$ ) from the source point [17]. Import-export routes also enhance the spread of FMD, potentially resulting in a highly damaging global economic impact.

FMD is one of the most economically important livestock diseases [7, 11]. Heavy import-export restrictions apply in countries that experience frequent FMD outbreaks [34]. Thus, the cost-benefit ratio of an investment business in FMD-affected animal species is greatly affected by frequent disease outbreaks. Due to its economic impact, FMD remains the greatest and most feared vesicular diseases in India [28]. In livestock production, the economic loss due to FMD can be calculated by considering, e.g., milk loss, disease-induced abortions and treatment costs. By the time the 2001 UK FMD outbreak had been stopped, the government had spent nearly GBP 3 billion on the operation of containing and cleaning up after the disease [37]. Recent outbreaks in Botswana include in 2002, 2005, 2006, 2007, 2011 and 2012 [3, 25, 29].

There is no cure for FMD [33, 40]. Infected animals usually recover to a healthcompromised status that renders them less profitable. Conventional control measures against FMD are movement restriction; public education; veterinary boundaries; quarantine; vaccination and culling (slaughtering animals in order to reduce the number of susceptible or infectious animals, and hence reduce spread of the disease [4]). Two basic forms of vaccination against foot and mouth disease are prophylactic vaccination (pre-outbreak: vaccination carried out prevent introduction of the disease) and ring vaccination (during an outbreak: carried out on farms neighboring infected farms). The Cedivac-FMD Double Oil Emulsion (DOE) vaccines (one of many types of FMD vaccines) confer a duration of immunity of at least 6 months in cattle, sheep and pigs $[10,15]$. Some vaccines can provide prolonged immunity for up to 12 months, depending on among others, the species affected and the virus serotype. 
Cattle which have recovered from infection with one of the seven serotypes of FMD remain protected against that serotype for up 6 months about 5 years, depending on the virus serotype [14]. Methods of culling include contagious premises $(\mathrm{CP})$ culling (slaughtering farms based on their proximity to infected farms) and infected premises (IP) culling (slaughtering infected farms).

The availability of data for the 2001 FMD outbreak in the United Kingdom allowed the development of validated epidemiological models, making it possible to explore impacts of various control measures [38]. For instance, Ref. [38] used an individual-farm based transmission probability model, capturing spatiality by describing the probability of infection as a function of the distance between susceptible farms and infection source (transmission kernel), and also explored impacts of ring vaccination strategies. Using a stochastic individual farm-based model, Ref. [23] explored impacts of either national prophylactic vaccination campaigns, or combinations of reactive (during outbreak) vaccination and culling.

Compartmental models have also been used to study the dynamics and control of foot and mouth disease. In compartmental models, the host population is comprised of subdivisions of the population called compartments such that the nature and time rates of transfer from one compartment to another are defined [8]. Each compartment represents the disease status of farms (e.g. susceptible, infectious or recovered). Compartmental models are sometimes referred to as mean-field approximations as they typically assume that members of the host population mix homogeneously [8]. Thus spatial spread of the disease is ne- glected [9], since it is assumed that an infectious farm is equally likely to infect any of the susceptible farms in the population. Ref. [30] adopt this approach to model the spread of FMD and impacts of vaccination, by dividing the population of farms into susceptible (S), vaccinated (V), latently infected (L) and infectious farms (I), and uses it to explore the impacts of births and deaths, culling, and vaccine waning.

Recently a number of foot and mouth disease transmission models have used using moment closure approximations (pair approximation models in particular) to capture spatiality implicitly. Ref. [32] design and analyze an SEI (susceptible, exposed but not infectious, infectious) pair approximation model of foot and mouth disease and explore impacts of IP culling and $\mathrm{CP}$ culling. They assume that the disease spreads on a network of farms represented by nodes (farms) and edges (links between farms). For many infectious diseases where spatiality is important for transmission and control, including foot and and mouth disease, spatially structured models may provide advantages over mean-field approximations such as conventional compartmental models $[31,5]$. Ref. [18] also present and analyze a pair approximation model of foot and mouth disease, employing data from the well-documented 2001 FMD out break in the United Kingdom, and explore impacts of ring culling and ring vaccination (both of which are applied during a single outbreak). In Ref. [18], the transmission rate is explicitly defined as a function of both local transmission between connected farms, and long range transmission due to transport since FMD virus can be transported to up to $60 \mathrm{~km}$ from the source point. 
While mean field approximations are formulated under an assumption that individuals in the host population mix homogeneously, moment closure approximations capture the spatial spread of diseases by modeling states of neighboring members of the host population. This technique provides information about the spatial distribution of disease states on a network and employs pairs, triples, quadruple, and other higher-order correlations as state variables of ordinary differential equations $[5,9]$. Each ordinary differential equation (also referred to as equation of motion for a state variable) measures the expected rate of change of a state variable by averaging all possible events affecting the state variable [39]. To do this, the first step is to write the equations of motion for the number of neighboring pairs of individuals or groups of individuals of a given state on a network; these equations will have terms involving triples [5]. The equations of motion for triples will involve quadruples while the equations of motion for quadruples will have terms involving five-order correlations. Essentially the procedure yields an infinite system of ordinary differential equations, each describing rates of change of state variables. However in order to solve the system analytically or using available computer software the system of equations needs to be finite. A closed, manageable system is obtained by truncating the hierarchy at some suitable level by a process known as moment closure $[6,5,39,21]$. When the system is closed at the level of pairs, it is referred to as pair approximations. Pair approximations models track down the dynamics of neighboring pairs of members of the host population, capturing the correlations that develop when two individuals interact
[5, 16]. Pair approximations also tend to be more analytically tractable than fully explicit network models.

Most models of FMD transmission are intended for epidemic settings, where control measures are designed to contain a single epidemic outbreak. However, FMD is an endemic problem in many countries. For example, in Botswana, FMD is endemic in some regions due to importation of FMD virus from wild African buffaloes and neighboring countries [3]. In endemic settings, long-term factors become important, such as waning of natural immunity, waning of vaccine immunity, and frequent disease re-introduction. Moreover, prophylactic and ring vaccination may become desirable control measures, under some circumstances. Despite the importance of such factors for FMD-endemic settings, they are not commonly included in spatial FMD transmission models. For example, to our knowledge there is no pair approximation FMD model that analyzes both ring and prophylactic vaccination. The same holds true for the impact of disease reintroduction. Our objective was to fill this gap in the literature by developing an SEIRV (susceptible, exposed but not infectious, infectious, recovered and vaccinated) pair approximation model of FMD to explore the impacts of prophylactic and ring vaccination, vaccine waning and loss of natural immunity as well as disease reintroduction from an external source, on the dynamics of foot and mouth disease in a fixed population of farms.

\section{Model}

The state variables of pair approximation models are of the form $[X Y]$, where $X$ 
and $Y$ represent the status of farms with respect to the disease so that $[X Y]$ is defined as the expected number of status $X$ and status $Y$ pairs at a given time, $t$. The dynamics of state variables of pair approximations models are governed by the master equation:

$$
\frac{d g(t)}{d t}=\sum r(\epsilon) \Delta g(\epsilon)
$$

where $g(t)$ is the state variable of interest, $r(\epsilon)$ is the rate of event $\epsilon$ and $\Delta g(\epsilon)$ is the change this event causes in $g(t)$.

As an example of pair approximation derivation, in Appendix (Appendix A) we derive the equation of motion for the number of susceptible-infectious, S-I pairs, $\frac{d[S I]}{d t}$, for an SEIRV (susceptible, exposed but not infectious, infectious, recovered and vaccinated) pair approximation model of FMD. We show how ring and prophylactic vaccination as well as vaccine waning and loss of disease induced immunity are incorporated and observe that

$$
\begin{aligned}
\frac{d[S I]}{d t}= & -\tau([I S I]+[S I])+\nu[S E] \\
& -\sigma[S I]-\psi_{r}([S I]+[I S I]) \\
& -\psi_{p}[S I]+\omega[I R]+\theta[I V],
\end{aligned}
$$

where $\tau, \sigma, \psi_{r}, \psi_{p}, \omega$ and $\theta$ are the transmission rate, recovery rate, rate of ring vaccination, rate of prophylactic vaccination, rate of loss of natural immunity and rate of vaccine waning, respectively. The number of $I-S-I$ triples enters the equation of motion for $I-S$ pairs because it is possible that transmission from one infected farm to a susceptible farm can destroy a $I-S$ pair consisting of that susceptible farm and a second infected farm, creating an $I-E$ pair in its place. The sign in front of the triple term is negative because an $I-S$ pair is disappearing. The latent period of FMD is given by $\nu^{-1}$, therefore $S-E$ is converted to $S-I$ (i.e. S-I bond is created), at rate $\nu$, leading to the term $+\nu[S E]$ on the RHS of this equation. The rest of the terms are developed in a similar manner.

If an equation of motion for $[I S I]$ is in turn formulated, it will involve quadruples and the hierarchy will go on to involve progressively higher order correlations. To truncate the hierarchy, we perform a moment closure approximation, a technique in which higher order correlations (order 3) are approximated in terms of lower order correlations (pairs and singletons). There exist various forms of moment closure approximations to the level of pairs which vary in the assumptions they make about the distribution of neighbors around a farm. Here we adopt the ordinary pair approximation (OPA) [32], and approximate the number of triples in terms of pairs and singletons, and the number of neighbors of a farm, $n$, as

$$
[X Y Z] \approx \frac{n-1}{n} \frac{[X Y][Y Z]}{[Y]}
$$

The ordinary pair approximation assumes that all individuals in the network have exactly $n$ contacts. The approximation maintains pair correlations between $X$ and $Y$, and between $Y$ and $Z$, but assumes higher order correlations between $X$ and $Z$ are negligible. In practice, $X$ and $Z$ could be correlated because they are directly connected, forming a triangle, or because $X$ and $Z$ have influenced one another via $Y$. The presence of triangles can be accounted for using a triangular approximation $[32,22]$. In contrast, in a mean field approach $[X Y]$ is approximated by $[X][Y]$ 
while $[X Y Z]$ would be approximated by $[X][Y][Z]$.

\subsection{Model equations}

$S, E, I, R$ and $V$ respectively, represent epidemiological states of the host popula- tion (farms): susceptible, exposed (not yet infectious), infectious, recovered and vaccinated. The full model equations are given by

$$
\begin{aligned}
& \frac{d[S]}{d t}=-\tau[S I]-\psi_{r}[S I]-\psi_{p}[S]+\omega[R]+\theta[V] \\
& \frac{d[E]}{d t}=\tau[S I]-\nu[E]-\psi_{r}[E I]-\psi_{p}[E] \\
& \frac{d[I]}{d t}=\nu[E]-\sigma[I] \\
& \frac{d[R]}{d t}=\sigma[I]-\omega[R] \\
& \frac{d[V]}{d t}=\psi_{r}[S I]+\psi_{r}[E I]+\psi_{p}[S]+\psi_{p}[E]-\theta[V] \\
& \frac{d[S S]}{d t}=-2 \tau[S S I]-2 \psi_{r}[S S I]-2 \psi_{p}[S S]+2 \omega[S R]+2 \theta[S V] \\
& \frac{d[S E]}{d t}=-\tau([I S E]-[S S I])-\nu[S E]-\psi_{r}([I S E]+[S E I])-2 \psi_{p}[S E]+\omega[E R]+\theta[E V] \\
& \frac{d[S I]}{d t}=-\tau([I S I]+[S I])+\nu[S E]-\sigma[S I]-\psi_{r}([S I]+[I S I])-\psi_{p}[S I]+\omega[I R]+\theta[I V] \\
& \frac{d[S R]}{d t}=-\tau[I S R]+\sigma[S I]-\psi_{r}[I S R]-\psi_{p}[S R]-\omega[S R]+\omega[R R]+\theta[R V] \\
& \frac{d[S V]}{d t}=-\tau[I S V]-\psi_{r}[I S V]-\psi_{p}[S V]+2 \psi_{r}[S S I]+2 \psi_{p}[S S]+\psi_{r}[S E I]+\psi_{p}[S E]+\omega[R V] \\
& +\theta[V V]-\theta[S V] \\
& \frac{d[E E]}{d t}=2 \tau[E S I]-2 \nu[E E]-2 \psi_{r}[E E I]-2 \psi_{p}[E E] \\
& \frac{d[E I]}{d t}=\tau([I S I]+[S I])+\nu([E E]-[E I])-\sigma[E I]-\psi_{r}([E I]+[I E I])-\psi_{p}[E I] \\
& \frac{d[E R]}{d t}=\tau[I S R]-\nu[E R]+\sigma[E I]-\psi_{r}[I E R]-\psi_{p}[E R]-\omega[E R] \\
& \frac{d[E V]}{d t}=\tau[I S V]-\nu[E V]-\psi_{r}[I E V]-\psi_{p}[E V]+\psi_{r}[I S E]+\psi_{p}[S E]+2 \psi_{r}[E E I]+2 \psi_{p}[E E] \\
& -\theta[E V] \\
& \frac{d[I I]}{d t}=2 \nu[E I]-2 \sigma[I I] \\
& \frac{d[I R]}{d t}=\sigma([I I]-[I R])+\nu[E R]-\omega[I R] \\
& \frac{d[I V]}{d t}=-\sigma[I V]+\nu[E V]+\psi_{r}([S I]+[I S I])+\psi_{p}[S I]+\psi_{r}([E I]+[I E I])+\psi_{p}[E I]-\theta[I V] \\
& \frac{d[R R]}{d t}=2 \sigma[I R]-2 \omega[R R]
\end{aligned}
$$




$$
\begin{aligned}
\frac{d[R V]}{d t} & =\sigma[I V]-\omega[R V]-\theta[R V]+\psi_{r}[I S R]+\psi_{p}[S R]+\psi_{r}[I E R]+\psi_{p}[E R] \\
\frac{d[V V]}{d t} & =2 \psi_{r}[I E V]+2 \psi_{r}[I S V]+2 \psi_{p}[S V]+2 \psi_{p}[E V]-2 \theta[V V]
\end{aligned}
$$

The factor two in the equations of motion pairs of the form $X X$ comes from the counting convention of same-status pairs, wherein pairs of type $\mathrm{X}-\mathrm{X}$ are counted twice. The ordinary pair approximation has been used to close the equations of motion.

During an outbreak of foot and mouth disease, transmission at rate $\tau$ takes place between an infectious and a susceptible farm, moving the latter to the exposed compartment. A farm stays in the exposed state for $\nu^{-1}$ days (latent period), after which it becomes infectious. The recovery rate (transition from infectious state to recovered compartment) is given by $\sigma$. Loss of natural immunity (diseaseinduced immunity) takes place at rate $\omega$, enabling transition of farms from $\mathrm{R}$ to $\mathrm{S}$ compartments. Prophylactic vaccination and ring vaccination at per capita rates $\psi_{p}$ and $\psi_{r}$, respectively, transfer vaccinated susceptible and exposed farms to the vaccinated compartment. The rate of loss of vaccine-induced immunity (vaccine waning) is given by $\theta$ (where farms lose protection from the vaccine, becoming susceptible again).

\subsection{The basic reproduction number}

The basic reproduction number, $R_{0}$, is defined as the expected number of secondary cases produced by a single infection in a completely susceptible population $[20,5,26,36]$. An epidemic is expected if $R_{0}>1$ and the infection is expected to die out if $R_{0}<1[36,19]$. In Appendix B we illustrate the derivation of a spatiallyoriented basic reproduction number for a pair approximation model without control measures :

$$
R_{0}=\frac{\beta(n-1)^{2}}{\sigma n\left[(n-1)+\left(\frac{\beta}{\nu}\right)\right]},
$$

where $\beta=\tau n, n$ is the number of neighboring farms. The basic reproduction number increases with the number of neighbors, $n$, on account of decreased opportunities for localized clustering of infected individuals to interfere with further transmission.

The basic reproduction number with ring vaccination as the only control measure is:

$$
\frac{m_{3} \tau\left(m_{1} \nu+m_{2} \psi_{r}\right)}{m_{3} \tau \psi_{r}+\left(m_{1} \nu+m_{2} \psi_{r}\right)\left(\frac{\sigma\left(m_{4} \nu+m_{5} \tau\right)}{v}+m_{5} \frac{\sigma \psi_{r} \nu}{\nu}\right)},
$$

where $m_{i}, i=1 \ldots 5$, are constants $\frac{n-1}{n}+1$, $\frac{n-1}{n} N q, n(n-1)^{2}, n(n-1)$ and $n^{2}$, respectively. In our model $n=4$ neighbors per farm, $N=40000$ farms and $q=1.5$ ( $q$ represents a ratio, $\frac{[E I]}{[E]}$ and converges to 1.5 as $t \rightarrow \infty$, on a square grid). Therefore $m_{1}=1.75, m_{2}=4.5 \times 10^{4}, m_{3}=36$, $m_{4}=12$ and $m_{5}=16$, respectively. The basic reproduction number with prophylactic vaccination only is:

$$
\frac{m_{3} \tau\left(m_{1} \nu+\psi_{p}\right)}{\left(m_{1} \nu+\psi_{p}\right)\left[\frac{\sigma\left(m_{4} \nu+m_{5} \tau\right)}{v}+\frac{m_{6} \nu+m_{5} \tau}{v} \psi_{p}\right]},
$$

$m_{6}=(n-1)^{2}=9$. The appearance of quadratic form of vaccination terms, $\psi_{r}$ and $\psi_{p}$, in the denominators of these expressions, guarantees that vaccination will 
decrease the basic reproduction number exponentially.

The basic reproduction number in the presence of prophylactic, $\psi_{p}$ and ring, $\psi_{r}$ vaccination (see Appendix B) is considerably more complicated and is given by:

$$
R_{0} \approx \frac{m_{7} m_{9}(n-1)^{3}+m_{7} m_{8} n(n-1)^{2}}{m_{10} n(n-1)^{2}+\left(m_{9} \frac{n-1}{n}+m_{8}\right)\left[m_{11} n(n-1)+m_{11} m_{12} n^{2}+m_{13}(n-1)^{2}+m_{12} m_{13} n(n-1)\right]},
$$

where $m_{7}=\tau, m_{8}=\nu+\psi_{r}+\psi_{p}$, $m_{9}=\nu+\psi_{r} N q, m_{10}=\tau \psi_{r}, m_{11}=\sigma$, $m_{12}=\frac{\tau+\psi_{r}}{\nu}, m_{13}=\psi_{p}$.

\subsection{Baseline parameters}

The baseline parameter values are defined in Table 1. Cattle, swine, sheep, goats and deer exhibit signs of clinical illness from FMD after an incubation period of about 2 to 14 days, [30]. The latent period of foot and mouth disease is 3.1 to 4.8 days in cattle [27]. Upon contact with the FMD virus, animals show clinical signs and are able to transmit the virus after 4 to 5 days [24]. Therefore we assume that the latent period is 4 days, thus $\nu=\frac{1}{4}=0.25 \mathrm{day}^{-1}$. Once in the infectious compartment, cattle show symptoms and remain infectious for about 7 to 8 days before they recover, [32]. Our baseline choice of the recovery rate is $\sigma=\frac{1}{7}=0.143 d a y^{-1}$.

Cattle which have recovered from infection with one of the seven serotypes of FMD are not immune to other serotypes but remain protected against the first serotype for a considerable period of time. Laboratory experiments show that the length of natural protection may range from 6 months to 5.5 years, depending on the serotype [14]. Using this observation as a guide, and considering the possibility for transmission of multiple serotypes in the same population in succession, our baseline choice of the duration of natural immunity is 6 months $(\approx 0.5$ years, or $\left.\omega=0.0056 d a y^{-1}\right)$, but we also explore scenarios of $\approx 1$ year $\left(\omega=0.0030 d_{a y}{ }^{-1}\right)$ and $\approx 2$ years $\left(\omega=0.0015\right.$ day $\left.^{-1}\right)$.

Cattle remain protected by FMD vaccine for up to 6 months [23], therefore $\theta=0.0056 d_{a y}{ }^{-1}$. We assume that per capita vaccination rates of $\psi_{p}=0.005$ per day and $\psi_{r}=0.005$ per day. In some countries, foot and mouth disease can spread across borders through animal movement or trade. In some parts of Botswana FMD is imported into Zimbabwe or South Africa resulting in a series of outbreaks almost every 2 years [29]. In our model simulations, disease is re-introduced into the population of farms every $\delta=800$ days (just over 2 years). The baseline transmission parameter is $\tau=0.6$ per day. In Appendix C we estimate this parameter value from the expression of the basic reproduction number (equation 5). We present all baseline parameters in Table 1. Finally, to partially account for the effects of stochastic fadeout in our deterministic framework, when the total number of infectious farms falls below 1 in the simulation (less than one infected farm left), the infection is forced to die out, so that any subsequent outbreaks are the result of disease re-importation. The population size is $N=40000$ farms. 


\section{Results}

We focus on the impact of loss of natural immunity, $\omega$, vaccine waning, $\theta$, and disease re-introduction frequency, $\delta$, on the number of outbreaks, average peak size of outbreaks, and cumulative number of infected farms over a given time period. We also consider impacts of prophylactic vaccination, $\psi_{p}$ and ring vaccination, $\psi_{r}$, on disease incidence, cumulative infections and the basic reproduction number, $R_{0}$.

Figure 1 shows the impact of loss of natural immunity, $\omega$, on disease reintroduction in the absence of control measures. For higher values of $\omega$, natural immunity is short-lived causing the pool of susceptible farms to be rapidly replenished. Hence, epidemics do not fade out and the infection prevalence converges to an endemic equilibrium (Figure 1a, b). In contrast, if $\omega$ is low, corresponding to longlived natural immunity, epidemics fade out and prevalence goes to zero after an outbreak. After some time, a subsequent disease introduction sparks a new outbreak. As a result, the epidemiology is characterized by sustained outbreaks every 3-5 years (Figure 1c, d). However, because the susceptible pool is relatively slow to replenish, not every disease re-introduction is successful in starting an outbreak, meaning that outbreaks are spaced further apart when natural immunity wanes more slowly (Figure $1 \mathrm{~d}$ versus $1 \mathrm{c}$ ).

As a result of interactions between timing of disease re-introduction and rate of natural immunity waning, the dependence of the size and number of outbreaks on the natural immunity waning rate is not linear (Figure 2). As the rate of waning natural immunity $\omega$ increases, so does the number of outbreaks over a fixed time window of 20 years, through a series of plateaus (Figure 2a). The average peak size of all outbreaks in this period of time also increases with $\omega$, although beyond a certain point, further increases in $\omega$ do not change the average peak size (Figure 2b).

The effectiveness of prophylactic vaccination, $\psi_{p}$ and ring vaccination, $\psi_{r}$ is strongly determined by the presence or absence of vaccine waning, $\theta$, and natural immunity waning, $\omega$ (Figure 3 ). If both vaccine immunity and natural immunity wane after 6 months, then a vaccination strategy with $\psi_{p}=0.005$ day $^{-1}$ and $\psi_{r}=0.01$ $d a y^{-1}$ fails to prevent the infection from becoming endemic (Figure 3a). However, if vaccines were to provide life-long immunity (i.e. $\theta=0$ ), vaccination prevents future outbreaks (except for a small second outbreak due to re-importation) and eventually leads to eradication (Figure $3 \mathrm{~b}$ ). Similarly, if natural immunity were lifelong (Figure 3c) or if both natural and vaccine immunity were lifelong (Figure 3d), the infection is eradicated even more quickly.

Both prophylactic and ring vaccination decrease the cumulative number of infected farms (see surface plots of cumulative infections versus $\psi_{r}$ and $\psi_{p}$, Figure 4 ). However, at similar per capita rates of vaccination, prophylactic vaccination appears to be more effective. This simply reflects the fact that more farms in total are vaccinated under prophylactic vaccination, for the same per capita vaccination rate. However, it may also reflect the fact that prophylactic vaccination is a preventive (preoutbreak) form of vaccination that can delay or prevent outbreaks altogether. Almost all of the variation in the effectiveness of prophylactic vaccination occurs in 
a range of values from $\psi_{p}=0$ to $\psi_{p}=0.005$ $d a y^{-1}$ (the upper limit corresponds to being able to vaccinate all farms after about 200 days).

Both vaccine waning, $\theta$, and natural immunity waning, $\omega$, affect the number of epidemics, average size of infection peaks and cumulative number of infected farms over a 20-year time window (Figure 5). The number of epidemics (Figure 5a), average infection peak (Figure 5b) and cumulative infections (Figure 5c) are highest when both natural immunity and vaccine waning rates take values close to the baseline parameters $\left(\omega=\theta=0.0055\right.$ day $^{-1}$, corresponding to lowest immunity duration considered here: 6 months). Generally speaking, the number of epidemics, average infection peak and cumulative infections depend more sensitively on changes in the rate of natural immunity waning $\omega$ than they do on the rate of vaccine waning $\theta$ (the height variation is greater along the $\omega$ axis than the $\theta$ axis).

When the frequency of disease importation $\delta$ is low (i.e. there is a long time interval between re-introductions), long periods of zero disease prevalence are interspersed with sharp epidemic outbreaks (Figure 6a). After an outbreak is finished, vaccination is sufficient to prevent FMD from becoming endemic, but not sufficient to prevent an outbreak after the next re-introduction. As the frequency of disease re-importation increases, outbreaks occur closer together, but they are smaller in magnitude (Figure 6a). However, despite the smaller magnitude of epidemic peaks, the cumulative incidence is nonetheless higher when re-introductions are close together (Figure $6 b$ ). Thus despite its capability to yield high-peak outbreaks, a lower rate of disease re-introduction produces fewer cumulative infections. Interestingly, when the rate of re-introduction is every 8 years, there is an outbreak every 8 years, but when the rate of re-introduction is only 3 years (respectively, 0.5 years), there is an outbreak only every $4-5$ years (respectively, 4 years), suggesting that re-introductions may prevent extinction of the pathogen in between outbreaks, thereby supporting endemic infection.

Both prophylactic vaccination, $\psi_{p}$, and ring vaccination, $\psi_{r}$ also have a significant impact on the $R_{0}$ value as computed from equation 6 (Figure 7). However, prophylactic vaccination reduces $R_{0}$ below 1 more quickly than ring vaccination. This is similar to the observation for the relative impact of both forms of vaccination on the cumulative number of infected farms (Figure 4). Prophylactic vaccination appears to perform better that ring vaccination because it does not only delay occurrence of outbreaks, but it also reduces the sizes of subsequent outbreaks and guarantees disease eradication if well administered.

\section{Discussion}

The occurrence of foot and mouth disease outbreaks has both economic and social impacts in many countries. Even though it has since been eradicated or is under control in most developed countries, the occurrence of foot and mouth disease in the developing world can, and does, create global impacts. To avoid introduction of FMD into disease-free countries, heavy import-export restrictions apply on trade of animals and their products. 
Here, we applied moment closure techniques to construct and analyze a pair approximation model of foot and mouth disease and explored impacts of loss of natural immunity, vaccine waning and disease re-introduction on infection dynamics and the basic reproduction number. At biologically plausible parameter values, both waning natural and vaccine immunity had significant impacts on the number and magnitude of outbreaks, cumulative number of infections, and feasibility of disease control. Hence, these factors are important to consider in any transmission model of FMD in an endemic country. These outcomes are somewhat more sensitive to the rate of waning natural immunity than the rate of waning vaccine immunity. We also found that vaccination is more effective if loss of natural immunity is low (i.e. recovered farms remain in 'recovered' compartment longer due to longer-lasting natural immunity). However, if farms lose natural immunity at a higher rate then it will be difficult to eliminate the disease. For the same per capita vaccination rate, prophylactic vaccination appears to be more effective than ring vaccination, partly because prophylactic vaccination better delays the occurrence of outbreaks, leading to smaller and less frequent subsequent outbreaks, and ensuring that the basic reproduction number stays below unity.

In most developing countries, vaccination capacity may be constrained by many factors including cost. Therefore as part of future work, this model can be modified to explore optimal and/or cost-effective prophylactic and ring vaccination strategies where control measures are forced to operate within constraints such as cost, availability of vaccine and manpower. Empirical data are generally lacking in FMD- endemic settings and this must also be addressed in order to better validate countryspecific FMD models.

The outcomes of this analysis provide spatially oriented insight into the dynamics of foot and mouth disease and its control in FMD endemic countries. The dependence of disease control effectiveness on loss of natural immunity and vaccine waning has not been well explored in the literature of foot and mouth disease models, but our analysis suggests that these factors are influential enough to necessitate inclusion in any mathematical model of FMD transmission and control in endemic countries. 
Table 1: Baseline parameters for our model

\begin{tabular}{ccc}
\hline \hline parameter & value & source \\
\hline Transmission rate, $\tau$ & $0.6 d a y^{-1}$ & {$[32]$} \\
Rate of moving from latent to infectious, $\nu$ & $0.25 d a y^{-1}$ & {$[24]$} \\
Rate of moving from infectious to recovered, $\sigma$ & $0.143 d a y^{-1}$ & {$[32]$} \\
Rate of loss of disease-induced immunity, $\omega$ & $0.0056 d a y^{-1}$ & {$[14]$} \\
Rate of loss of vaccine-induced immunity, $\theta$ & $0.0056 d a y^{-1}$ & {$[14,23]$} \\
Rate of ring vaccination, $\psi_{r}$ & $0.005 d a y^{-1}$ & assumption \\
Rate of prophylactic vaccination, $\psi_{p}$ & $0.005 d a y^{-1}$ & assumption \\
Frequency of disease re-introduction, $\delta$ & every 800 days & {$[29]$} \\
\hline
\end{tabular}


Figure 1: Time series of the number of infectious farms where rates of natural immunity waning $\omega$ are $0.0055 d_{d a y^{-1}}$ (a), $0.0042 d a y^{-1}$ (b), $0.0014 d a y^{-1}$ (c) and $0.00055 d a y^{-1}$ (d). $\psi_{p}, \psi_{r}=0$ and all other parameters are as in Table 1 .

Figure 2: Number of outbreaks (a) and average infection peak (b) of outbreaks over a 20 year period versus rate of natural immunity waning, $\omega . \psi_{p}=\psi_{r}=0 d a y^{-1}$ and all other parameters are as in Table 1.

Figure 3: Time series for the number of infectious farms where $\omega=\theta=0.0055$ day $^{-1}$ (a), $\omega=0.0055$ $d a y^{-1}$ and $\theta=0(\mathrm{~b}), \omega=0$ and $\theta=0.0055 \operatorname{day}^{-1}(\mathrm{c})$ and $\omega=\theta=0$ (d). $\psi_{p}=0.005 \mathrm{day}^{-1}, \psi_{r}=0.01$ $d a y^{-1}$ and all other parameters are as in Table 1.

Figure 4: Cumulative number of infectious farms over 5 years as a function of prophylactic vaccination, $\psi_{p}$ and ring vaccination, $\psi_{r}$ vaccination. All other parameters are as in Table 1.

Figure 5: Number of outbreaks (a), average infection peak (b) and cumulative infections (c) over a 20 year period versus rate of natural immunity waning, $\omega$ and vaccine waning, $\theta . \psi_{p}=\psi_{r}=0.001$ day $^{-1}$ and all other parameters are as in Table 1.

Figure 6: Time series for the number of infectious farms for varying rates of disease re-introduction (a) and cumulative number of infected farms versus disease re-introduction (b) over a 20 year period. $\psi_{p}=0.00005 d a y^{-1}, \psi_{r}=0.0005 d a y^{-1}, \theta=\omega=0.0014 d a y^{-1}$ and all other parameters are as in Table 1.

Figure 7: The basic reproduction number, $R_{0}$ versus prophylactic vaccination, $\psi_{p}$ and ring vaccination, $\psi_{r}$. All other parameters are as in Table 1 . 


\section{Appendix A. Derivation of the equation of motion for [SI]}

We proceed with the derivation of a SEIRV pair approximation model of FMD as follows, using a similar approach to Refs. [5] and [22]. We demonstrate the derivation of the equation of motion for [SI].

The dynamics of [SI] are governed by the equation:

$\frac{d g(t)}{d t}=\sum r(\epsilon) \Delta g(\epsilon)$,

where $g(t)$ is the state variable of interest (i.e. [SI]), $r(\epsilon)$ is the rate of event $\epsilon$ and $\Delta g(\epsilon)$ is the change this event causes in $g(t)$ (i.e. [SI]).

As the disease progresses, [SI] is affected by the by the following events.

Infection of the susceptible farm by the infectious farm in the S-I edge converts $\mathrm{S}$ into E, i.e. $S I \mapsto E I$, where $\mapsto$ means 'transformed to'. This adds $-\tau[S I]$ into the equation of motion for [SI]. The negative sign,-, in the coefficient is a result of this event 'destroying' S-I edges.

Infection of the susceptible farms 'from the left' in a triple I-S-I, i.e. $I \leftrightarrow S I$ gives rise to $S I \mapsto E I$, contributing the term $-\tau[I S I]$ into the equation of motion for [SI].

Latent period is $\frac{1}{\nu}$, therefore $S E \mapsto S I$ and the process 'creates' SI (hence positive coefficient). Thus we add $\nu[S E]$ into the equation of motion for [SI].
A infectious farm recovers at rate, $\sigma$, therefore $S I \mapsto S R$ contributing $-\sigma[S I]$ into the equation of motion for [SI].

Ring vaccination (defined as vaccination of exposed and susceptible farms that have links with infected farms) in the susceptible farm in a pair S-I, at rate $p s i_{r}$ converts SI to RI and adds $-\psi_{r}[S I]$ to $\frac{d[S I]}{d t}$.

Ring vaccination in the susceptible farm in a triple I-S-I, at rate $p s i_{r}$ converts SI to $\mathrm{RI}$ and adds $-\psi_{r}[I S I]$ to $\frac{d[S I]}{d t}$.

Prophylactic vaccination at rate, $\psi_{p}$ (defined as vaccination of all susceptible and exposed farms whether or not they are connected to infectious farms) of the susceptible farms in S-I, adds $-\psi_{p}[S I]$.

A recovered farm in an I- $R$ pair loses natural immunity at rate $\omega$ to form an S-I pair, thus adding $\omega[I R]$ to $\frac{d[S I]}{d t}$.

A vaccinated farm in an $\mathrm{I}-\mathrm{V}$ pair loses vaccine protection at rate $\theta$ to form an S-I pair, thus adding $\theta[I V]$ to $\frac{d[S I]}{d t}$.

Therefore the equation of motion for [SI] is

$\frac{d[S I]}{d t}=-\tau([I S I]+[S I])+\nu[S E]-\sigma[S I]-$ $\psi_{r}([S I]+[I S I])-\psi_{p}[S I]+\omega[I R]+\theta[I V]$.

\section{Appendix B. Derivation of the the basic reproduction number}

We derive the expression of the basic reproduction number for a pair approximation model of foot and mouth disease without control measures. The equations of 
motion for the number of exposed and infectious farms are important in the derivation of the basic reproduction number:

$\frac{d}{d t}[E]=\tau[S I]-\nu[E]$ and

$\frac{d}{d t}[I]=\nu[E]-\sigma[I]$.

An epidemic is expected if $\frac{d}{d t}[E]+\frac{d}{d t}[I]>0$ and the disease is expected to die out if $\frac{d}{d t}[E]+\frac{d}{d t}[I]<0$. Using the correlation function between susceptible and infectious farms, $C_{S I}=\frac{N}{n} \frac{[S I]}{[S][I]}$, we re-write the equation of motion for the number of exposed farms as

$\frac{d}{d t}[E]=\frac{\beta}{N}[S][I] C_{S I}-\nu[E]$, where $\beta=\tau n$.

At the beginning of an epidemic almost all farms are susceptible, i.e. $[S] \approx N$. Therefore we simplify the equation of motion for the number of exposed farms further:

$\frac{d}{d t}[E]=\beta[I] C_{S I}-\nu[E]$.

An epidemic is expected if $\frac{d}{d t}[E]+\frac{d}{d t}[I]=$ $\beta[I] C_{S I}-\nu[E]+\nu[E]-\sigma[I]>0$, i.e.

$\beta[I] C_{S I}-\sigma[I]>0$.

Thus there will be an epidemic if $\frac{\beta}{\sigma} C_{S I}>1$. Therefore the expression of the basic reproduction number is

$$
R_{0}=\frac{\beta}{\sigma} C_{S I}
$$

The correlation between susceptible and infectious farms, $C_{S I}$ is not constant but it changes from $C_{S I} \approx 1$ at the beginning of the infection, decreasing as more individuals become infected [5]. An increase in the number of infected farms leads to reduction of the infection rate in the long run, and at this point the epidemic may fade out. This is a local minimum of $C_{S I}$. The dynamics of the disease at this point are important in deriving an explicit form of the basic reproduction number. Therefore we seek $C_{S I}^{m i n}$, the local minimum value of $C_{S I}$, obtained by solving $\frac{d}{d t} C_{S I}=0$.

The derivative of the correlation function between the number of susceptible and infectious farms is given by

$\frac{d}{d t} C_{S I}=\frac{N}{n} \frac{1}{[S][I]} \frac{d}{d t}[S I]+\frac{N}{n}[S I] \frac{d}{d t} \frac{1}{[S][I]}$.

This expression is equivalent to:

$\frac{d}{d t} C_{S I}=\frac{N}{n} \frac{1}{[S][I]} \frac{d}{d t}[S I]+C_{S I}\left(-\frac{1}{[I]} \frac{d}{d t}[I]-\right.$
$\left.\frac{1}{[S]} \frac{d}{d t}[S]\right)$.

Substituting the equations of motion for $[S I],[I]$ and $[S]$, into the equation above, applying the ordinary pair approximation to express the number of triple as pairs and singletons, and noting that at the initial stages of the disease there are no recovered farms (i.e. $[R] \approx 0$ ), yields

$\frac{d}{d t} C_{S I}=-\tau(n-1) \frac{[I]}{N} C_{S I}^{2}-\tau C_{S I}+\nu \frac{[E]}{[I]} C_{S E}-$ $\nu \frac{[E]}{[I]} C_{S I}+\tau n \frac{[I]}{N} C_{S I}^{2}$.

Note that at the initial stages of the infection $[S] \approx N$; a simple analysis of terms involving $\frac{[I]}{N} C_{S I}^{2}$ shows that they are too small to have significant impact on the correlation between susceptible and infectious farms. We let $\frac{[I]}{N} C_{S I}^{2} \rightarrow 0$ so that the equation of motion for $C_{S I}$ becomes

$\frac{d}{d t} C_{S I}=-\tau C_{S I}+\nu \frac{[E]}{[I]} C_{S E}-\nu \frac{[E]}{[I]} C_{S I}$.

To solve for $C_{S I}^{\min }$, explicitly, we need simpler representation for $C_{S E}$ and $\frac{[E]}{[I]}$. 
In a network where there are no 'triangles', when a susceptible farm becomes exposed, this newly exposed farm inherits the neighborhood of the susceptible [32, 22]. Thus

$C_{S E} \approx \frac{(n-1)}{n} C_{S S}$

Adopting arguments by [32] we assume further that the host population space is such that $C_{S S} \approx 1$, so that

$C_{S E} \approx \frac{(n-1)}{n}$.

The process of a farm moving from the exposed to the infectious state implies that the farm now inherits a fraction $(n-1) / n$ of the neighborhood it had previously $[32,22]$. Thus

$\frac{[E]}{[I]} \approx\left(\frac{n-1}{n}\right)$.

Therefore the equation of motion for $C_{S I}$ is $\frac{d}{d t} C_{S I}=-\tau C_{S I}+\nu \frac{(n-1)}{n} \frac{(n-1)}{n}-\nu \frac{(n-1)}{n} C_{S I}$.

We obtain $C_{S I}^{\min }$ by letting the left hand side of the equation above equal zero and solve for $C_{S I}$. It follows that

$C_{S I}^{\min }=\frac{(n-1)^{2}}{n\left[(n-1)+\left(\frac{\beta}{\nu}\right)\right]}$ where $\beta=\tau n$.

But $R_{0}=\frac{\beta}{\sigma} C_{S I}^{\min }$, therefore

$$
R_{0}=\frac{\beta(n-1)^{2}}{\sigma n\left[(n-1)+\left(\frac{\beta}{\nu}\right)\right]}
$$

where $\beta=\tau n$. Under mean field approximation, the corresponding expression of the basic reproduction number is $R_{0}=\frac{\tau}{\sigma}$. This overestimates the true $R_{0}$ in a spatially structured population of farms because it does not take into account the slowing effects of spatially localized transmission [5]. On the other hand, the expression of the basic reproduction number derived under moment closure techniques provides a better estimation of the true value of $R_{0}$ because of the pair approximations' capacity to capture the time evolution of local spatial structure. Here transmission can only take place between neighboring farms.

\section{Appendix C. Derivation of the transmission parame- ter, $\tau$}

We derive the baseline transmission rate, $\tau$ from the expression of the basic reproduction number, equation 5 . Substituting $n=4, \beta=\tau n=4 \tau, \nu=1 / 4$ and $\sigma=1 / 7$ into this expression yields

$R_{0}=\frac{63 \tau}{3+16 \tau}$.

Changing the subject of this formula to $\tau$ gives

$\tau=\frac{3 R_{0}}{63-16 R_{0}}$.

$\tau$ takes positive values only when $R_{0}>0$ and $63-16 R_{0}>0$. Thus transmission will take place when

$0<R_{0}<\frac{63}{16}$, i.e. $0<R_{0}<3.9375$.

The corresponding baseline choice of $\tau$ should be based on the choice of $R_{0}$ in this interval.

Also on a square grid, if recovery rate, $\sigma=0$, an infected farm can infect no more than $n-1$ neighbors, none of whom ever recover (the farm became infected through 
one of its neighbors, so no more than $n-1$ neighbors can be susceptible) [5]. Thus $R_{0} \leq n-1$. If infectious farms recover (to recovered compartment), i.e. $\sigma>0$, then the susceptible denominator is still at most $n-1$, i.e. $R_{0} \leq n-1$.

Thus if each farm has $n=4$ neighbors, then $R_{0} \leq n-1=3$, for all $\tau$ and $\sigma$.
Therefore on a squares-grid torus, an infected farm can infect at most 3 of its 4 neighbors.

If $R_{0}=1$, then $\tau=\frac{3 \times 1}{63-16 \times 1}=\frac{3}{47} \approx 0.064$;

If $R_{0}=2$, then $\tau=\frac{3 \times 2}{63-16 \times 2}=\frac{6}{31} \approx 0.194$;

If $R_{0}=3$, then $\tau=\frac{3 \times 3}{63-16 \times 3}=\frac{9}{15} \approx 0.600$.

Our baseline choice of the transmission rate is $\tau=0.600$. 


\section{References}

[1] A. Alonso, M.A. Martins, M.D. Gommes, R. Allende, and M.S. Sondahl. Foot and mouth disease viral typing by complement fixation and enzyme-linked immunosorbent assay monovalent and polyvalent antisera. J Ver Diagn Invest, 4:249-253, 1992.

[2] E.C. Anderson, J. Anderson, and J. Doughty. The foot and mouth disease subtype variants in kenya. J Hyg (London), 72(2):237-244, 1974.

[3] E.K. Baipoledi, G.Matlho, M. Letshwenyo, M. Chimbombi, E.K. Adom, M.V. Raborokgwe, and J.M.K. Hyera. Re-emergence of foot and mouth disease in botswana. The Veterinary Journal, 168:93-99, 2004.

[4] S.J. Barteling. Development and performance of inactivated vaccines against foot and mouth disease. Rev. sci. tech. Off, 21(3):577-588, 2002.

[5] C.T. Bauch. The spread of infectious diseases in spatially structured populations: an invasory pair approximation. Mathematical Biosciences, 198:217-237, 2005.

[6] C.T. Bauch and D.A. Rand. A moment closure model for sexually transmitted disease transmission through a concurrent partnership network. The Royal Society, 267:2019-2027, 2000.

[7] G.J. Belsham, S.M. Jamal, K. Tk $\phi$ rneh $\phi$ j, and A. B $\phi$ tner. Rescue of foot-and-mouth disease viruses that are pathogenic for cattle from preserved viral rna samples. PLoS ONE, 6(1):1-10, 2011.

[8] F. Brauer. Some simple epidemic models. Mathematical Biosciences and Engineering, $3(1): 1-15,2006$.

[9] K. Bunwong. A new approach to pair approximation method for spatial model in ecology. WSEAS Transactions on Mathematics, 9(10):768-777, 2010.

[10] G. Chenard, P. Selman, and A. Dekker. Cedivac-fmd can be used according to a marker vaccine principle.

[11] E.M. Cottam, J. Wadsworth, A.E. Shaw, R.J. Rowlands, L. Goatley, S. Maan, N.S. Maan, P.P.C. Mertens, K. Ebert, Y. Li, E.D. Ryan, N. Juleff, N.P. Ferris, J.W. Wilesmith, D.T. Haydon, D.P. King, D.J. Paton, and N.J. Knowles. Transmission pathways of foot-and-mouth disease virus in the united kingdom in 2007. PLoS Pathogens, 4(4):1-8, 2008.

[12] G. Davies. Foot and mouth disease. Research in Veterinary Science, 73:195-199, 2002.

[13] Y. Ding, H. Chen, J. Zhang, J. Zhou, L. Ma, L. Zhang, Y. Gu, and Y. Liu. An overview of control strategy and diagnostic technology for foot-and-mouth disease in china. Virology Journal, 10(78):1-6, 2013. 
[14] T.R. Doel. Natural and vaccine-induced immunity to foot and mouth disease: the prospects for improved vaccines. Rev. sci. tech. Off. int. Epiz, 15(3):883-911, 1996.

[15] J. Domenech, J. Lubroth, and K. Sumption. Immune protection of animals: the examples of rinderpest and foot and mouth disease. Journal of Comparative Pathology, 142:120-124, 2010.

[16] S.P. Ellner. Pair approximation for lattice models with multiple interaction scales. Journal of Theoretical Biology, 210:435-447, 2001.

[17] M. Enserink. Barricading u.s. boarders against a devastating disease. Science, 291:2298, 2001.

[18] N.M. Ferguson, C.A. Donnelly, and R.M. Anderson. The foot and mouth epidemic in great britain: pattern of spread and impact of interventions. Science, 292:1155-1160, 2001.

[19] J.M. Heffernan, R.J. Smith, and L.M. Wahl. Perspectives an the basic reproduction ratio. Journal of the Royal Society, 2(4):281-293, 2005.

[20] H.W. Hethcote. The mathematics of infectious diseases. Society for Industrial and Applied Mathematics, 42(4):599-653, 2000.

[21] D. Hiebeler. Moment equations and dynamics of a household sis epidemiological model. Bulletin of Mathematical Biology, 68:1315-1333, 2006.

[22] M.J. Keeling, D.A. Rand, and A.J. Morris. Correlation models for childhood epidemics. Proceedings of The Royal Society of London, B 264:1149-1156, 1997.

[23] M.J. Keeling, M.E.J. Woolhouse, R.M. May, G. Davies, and B.T. Grenfel. Modeling vaccination strategies against foot and mouth disease. Nature, 421:136-142, 2003.

[24] M.J. Keeling, M.J. Woolhouse, D.J. Shaw, L. Matthews, M. Chase-Topping, D.T Haydon, S.J. Cornell, J. Kappey, J. Wilesmith, and B.T. Granfell. Dynamics of the 2001 uk foot and mouth disease: stochastic dispersal in a heterogeneous landscape. Science, 294(5543):813-817, 2001.

[25] M. Letshwenyo, M. Fanikiso, and M. Chimbombi. The control of foot and mouth disease in botswana: special reference to vaccination. Dev Biol (Basel), 119:403-413, 2004.

[26] J. Li, D. Blakeley, and R.J. Smith? The failure of $r_{0}$. Computational and Mathematical Methods in Medicine, 12:1-17, 2011.

[27] F. Mardones, A. Perez, J. Sanchez, M. Alkhamis, and T. Carpenter. Parameterization of the duration of infection stages of serotype o foot-and-mouth disease virus: an analytical review and meta-analysis with application to simulation models. Vet Res, $41(4): 45,2010$. 
[28] L. Matthew and D.G. Menon. Economic impact of fmd in chazhoor panchayath. Veterinary World, 1(1):5-6, 2008.

[29] M. Mokopasetso and N. Derah. Recent outbreaks of foot and mouth disease in botswana and zimbabwe. Special Issues, 23:8-12, 2005.

[30] S. Mushayabasa, C.P. Bhunu, and M. Dhlamini. Impact of vaccination and culling on controlling foot and mouth disease: a mathematical modeling approach. World of Journal Vaccines, 1:156-161, 2011.

[31] P.E. Parham and N.M. Ferguson. Space and contact networks: capturing of the locality of disease transmission. Journal of Royal Society, 3:483-493, 2005.

[32] P.E. Parham, B.K. Singh, and N.M. Ferguson. Analytical approximation of spatial epidemic models of foot and mouth disease. Theoretical Population Biology, 72:349$368,2008$.

[33] H.J. Pharo. Foot and mouth disease: an assessment of the risks facing new zealand. New Zealand Veterinary Journal, 50(2):46-55, 2002.

[34] A.N. Rae, C. Nixon, and P. Gardiner. Foot-and-mouth disease and trade restrictions: Latin america access to pacific rim beef. The Australian Journal of Agriculture and Resource Economics, 43(4):479-500, 1999.

[35] N.M. Rweyamamu. Foot and mouth disease control strategies in africa. Preventative Veterinary Medicine, 2:329-340, 1984.

[36] P.H.T. Schimit and L.H.A. Monteiro. On estimating the basic reproduction number in distinct stages of a contagious disease spreading. Ecological Modelling, 240(2012):156160, 2012.

[37] D. Thompson, P. Muriel, D. Russell, P. Osborne, A. Bromley, M. Rowland, S. CreighTyte, and C. Brown. Economic costs of the foot and mouth disease outbreak in the united kingdom in 2001. Rev Sci Tech, 21(3):675-687, 2002.

[38] M.J. Tildesley, N.J. Savill, D.J. Shaw, R. Deardon, S.P. Brooks, M.E.J. Woolhouse, B.T. Grenfell, and M.J. Keeling. Economic costs of the foot and mouth disease outbreak in the united kingdom in 2001. Rev Sci Tech, 21(3):675-687, 2001.

[39] M. van Baalen. Pair approximations for different spatial geometries. The geometry of ecological interactions: simplifying spatial complexity, pages 359-387, 2000.

[40] U. Wernery and J. Kinne. Foot and mouth disease and similar virus infections in camelids: a review. Rev. sci. tech. Off. int. Epiz., 31(3):907-918, 2012. 